\title{
Measurement of lattice rotations and internal stresses in over one hundred individual grains during a stress-induced martensitic transformation
}

\author{
Younes El Hachi ${ }^{1,2}$, Benoit Malard ${ }^{1, a}$, Sophie Berveiller ${ }^{2}$, and Jonathan Wright ${ }^{3}$ \\ ${ }^{1}$ CIRIMAT, 4 Allée Emile Monso 31030 Toulouse - France \\ ${ }^{2}$ Arts et Métiers ParisTech, LEM3,UMR CNRS 7239, 4, rue Augustin-Fresnel, 57070 Metz - France \\ ${ }^{3}$ E.S.R.F - 71 Avenue des Martyrs - 38000 Grenoble - France
}

\begin{abstract}
To better understand the properties of polycrystals at a microscopic scale during cyclic mechanical loading we have measured the relationship between grain orientations, their positions inside the sample and their internal stresses. In this work, in-situ 3DXRD technique was performed on over hundred grains during the stress-induced martensitic transformation in a $\mathrm{Cu}-\mathrm{Al}-\mathrm{Be}$ shape memory alloy. Information about the position, orientation, and stress field was obtained for each austenitic grain. These results have been used to develop a procedure that allows automatic processing for a large number of grains, matching them during loading and leads to a quantitative stress field. A strong heterogeneity of stress state between the grains at the surface and in the volume is evident.
\end{abstract}

\section{INTRODUCTION}

The bulk properties of Shape Memory Alloys (SMA), such as the macroscopic stress-strain during the superelasticity effect, have been widely studied at the macroscopic scale. For better understanding the behavior and modeling it, it is necessary to access information at lower microstructural levels. At mesocopic scale, the average behavior of each phase, austenite and martensite, can be measured by X-rays or neutron diffraction: the stress-strain state depends strongly on the crystallographic orientation of the grains [1]. However, few experimental data are available at the grain scale. Some experiments have been performed on single crystals [2] or on coarse grains materials [3]. In Cu-based alloys, a factor 3 between the critical shear stresses was observed. However these results cannot be easily extended to the case of polycrystals where the individual grains are embedded in the bulk material as they do not take into account grain interactions. To obtain such data it is necessary to validate nondestructive analyzes for accessing surface as well as volume data. The 3DXRD technique [4-5] can measure such volumetric data during in-situ tests. It has been used to study lattice rotations of individual grains in aluminium alloys [6] and in $\mathrm{Cu}-\mathrm{Al}-$ Be SMA [7], phase transformation kinetics in steels [8-9].

In this paper we describe the development and the methodology to simultaneously track the stress tensors of hundreds of individual grains inside a polycrystalline shape memory alloy during an in-situ tensile test. In the following, we describe the technical 3DXRD, the methodology of the image treatment; then the results are presented and discussed.

\section{THE 3DXRD TECHNIQUE}

\section{1 Experimental set-up}

The 3DXRD microscopy has been developed to perform structural and crystallographic investigations in bulk materials, for each individual grain of a polycrystalline sample [4-5]. We used the 3DXRD microscope at beamline ID11 of the European Synchrotron Radiation Facility (ESRF, Grenoble, France) in transmission mode. The energy was $80 \mathrm{keV}(\lambda=0.155 \AA)$, corresponding to a penetration depth in copper of $1.5 \mathrm{~mm}$. The whole experimental set-up is shown figure 1 .

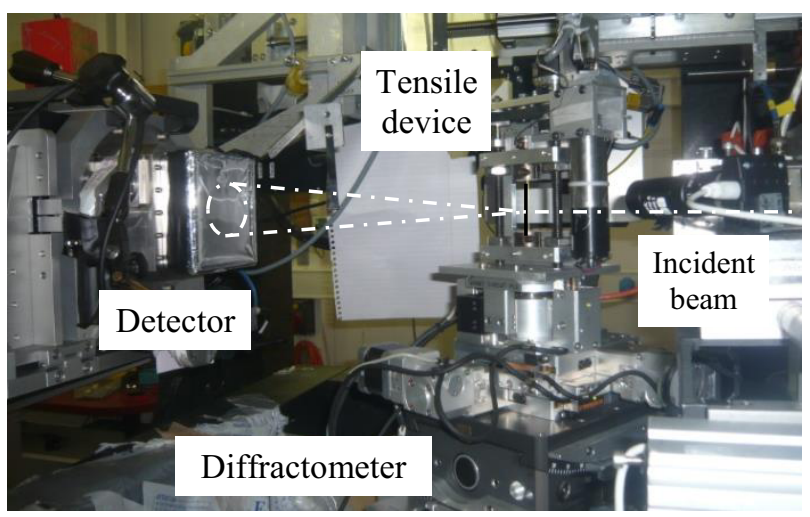

Figure 1. The 3DXRD set-up with the tensile device on the ID11 beamline at ESRF. Dashed lines show where the x-ray beam diffracts.

In order to follow the stress-induced transformation, an in-situ tensile device was installed on the beamline with

\footnotetext{
${ }^{\mathrm{a}}$ benoit.malard $@$ ensiacet.fr
}

This is an Open Access article distributed under the terms of the Creative Commons Attribution License 4.0, which permits unrestricted use distribution, and reproduction in any medium, provided the original work is properly cited. 
the tensile axis was vertical. The beam size was chosen to ensure that the whole width of the specimen remains illuminated by X-rays during rotation. The beam was $1 \mathrm{~mm}$ high and $1.6 \mathrm{~mm}$ wide for a specimen diameter of $1.2 \mathrm{~mm}$. To ensure that the illuminated zone is always the same while loading, two nickel wires were fixed on the specimen; after each loading increment, the center of the beam was aligned to be equidistant between these wires. Therefore, the illuminated zone is always centered at the same position in the sample.

Diffraction patterns were recorded on a CCD detector with an effective pixel size of $50 \mu \mathrm{m}$ and placed at a distance of $294 \mathrm{~mm}$ from the sample.

We used the rotation method as described by Lauridsen et al. [10-11]: images were acquired while rotating the specimen around the $\omega$-axis that was parallel to the tensile axis. At each equidistant step $\Delta \omega$ of $0.3^{\circ}$, a diffraction pattern was recorded. A pair of $114^{\circ} \omega$-ranges were used which corresponded to the incident beam entering the front and back of the load frame device $\left(228^{\circ}\right.$ in total). This corresponds to 760 images per load step and required a measurement time of 22 minutes.

\subsection{Indexing patterns}

Each scan is composed of 760 images. Grains are characterized by a collection of diffraction spots which can be indexed on a single lattice. For each observed spot on the images the scattering vectors were computed and these were grouped into hkl reflection families using the cell parameters input from the known crystallographic structure for austenite. Trial orientations for potential grains in the sample were found from pairs of observed spots which are separated by the expected angles for their hkl families. Orientations were retained when they predict a large number of diffraction spots. The center of mass positions (CM) of the grains and their orientations were then optimized. The calculations were all performed using the ImageD11 [12] software package. The grain volumes were estimated from the diffracted intensity.

A new development was needed to match the grains between two loading points so as to follow the same grain during the whole loading experiment. A calculation code was developed to analyze grains maps obtained from ImageD11 in order to relate them and be able to follow each individual grain during the mechanical cycle. Two solutions can be used: identifying grains by their orientation or by their spatial position in the specimen. The algorithm searches for the grains with the nearest position and/or the nearest crystallographic orientation of one grain between two maps (i.e. two loading points); in other words, two grains in two different maps are considered to be the same if the misorientation and the distance between their $\mathrm{CM}$ are below threshold values. We have matched grain maps considering both factors separately or together; the final grain pairing was always the same, showing the effectiveness of the algorithm for these data.

The elastic strain tensor of each grain was calculated from the measurement of the cell parameters given by Image D11. It is given by:

$$
\boldsymbol{\varepsilon}=1 / 2\left[\mathbf{A}\left(\mathbf{A}_{0}\right)^{-1}+\left(\mathbf{A}\left(\mathbf{A}_{0}\right)^{-1}\right)^{\mathrm{T}}\right]-\mathbf{I}
$$

where $\mathbf{A}$ et $\mathbf{A}_{\mathbf{0}}$ are respectively the cell parameter matrix of the deformed and undeformed grain [13].

$$
\mathbf{A}=\left(\begin{array}{ccc}
a & b \cos (\gamma) & c \cos (\beta) \\
0 & b \sin (\gamma) & c \sin (\beta) \cos \left(\alpha^{*}\right) \\
0 & 0 & c \sin (\beta) \sin \left(\alpha^{*}\right)
\end{array}\right)
$$

The stress tensor is then deduced using Hooke's law:

$$
\sigma_{\mathrm{ij}}=\mathrm{C}_{\mathrm{ijkl}} \times \varepsilon_{\mathrm{kl}}
$$

In our case, the non deformed $\left(\mathrm{d}_{0}\right)$ cell parameter matrix was defined for each individual grain of the sample from the initial diffraction pattern. This assumes the initial state was free from residual stresses.

\section{RESULTS}

\subsection{The Cu-Al-Be alloy}

We studied a $\mathrm{Cu}-\mathrm{Al}-\mathrm{Be}$ alloy whose chemical composition (in $\mathrm{wt}^{\%} \%$ ) is: $11.5 \% \mathrm{Al} ; 0.5 \% \mathrm{Be}$; and $88.0 \% \mathrm{Cu}$. The alloy was betatized at $760^{\circ} \mathrm{C}$ then water quenched. At room temperature, it is fully austenitic; its transformation start temperature $\mathrm{M}_{\mathrm{s}}$ was measured by $\mathrm{DSC}$ and is around $-100^{\circ} \mathrm{C}$. The specimen was cylindrical with a diameter of $1.17 \mathrm{~mm}$, cut from a drawn wire. The microstructure is shown figure 2. The mean grain size, determined by optical microscopy, is around $150 \mu \mathrm{m}$.

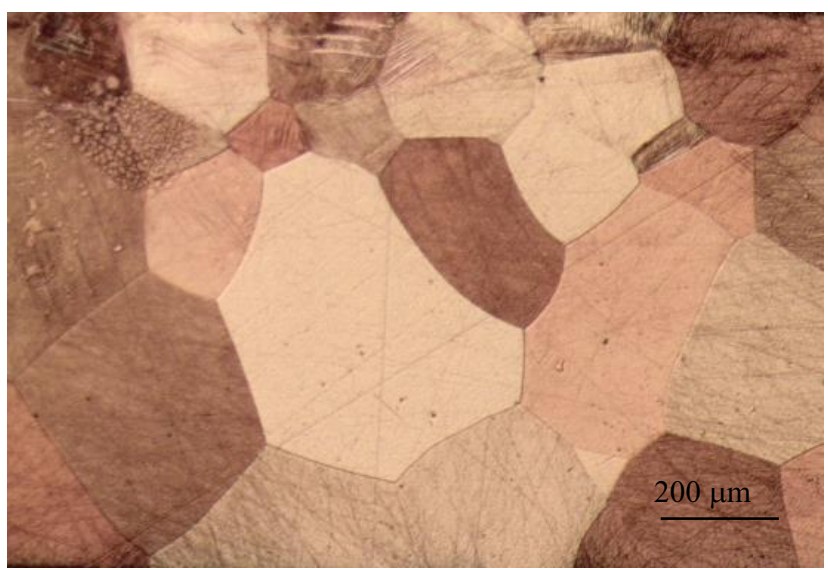

Figure 2. Microstructure of the studied alloy (optical microscopy).

Measurements were performed during an in-situ tensile loading cycle at room temperature. The macroscopic strain-stress curve of the alloy is given 
figure 3. A classical superelastic behavior of is observed; the macroscopic critical transformation stress is around $300 \mathrm{MPa}$. Diffraction patterns were recorded at different loading points, labeled on figure 3 , in the elastic domain and in the transformation one.

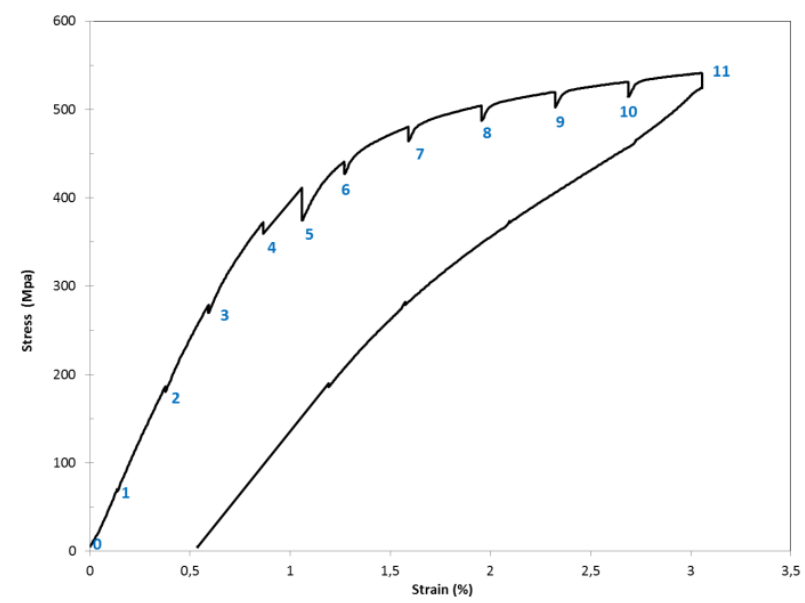

Figure 3. Macroscopic stress-strain curve at room temperature and 3DXRD measurents points.

As mentioned previously, the stress tensor is calculated from elastic strain measured by diffraction. Although if both martensite and austenite diffracted, we have only studied the evolution in the cubic austenite phase. The elastic constants of the austenite phase are: $\mathrm{C}_{11}=141.6 \mathrm{GPa} ; \mathrm{C}_{12}=127.4 \mathrm{GPa} ; \mathrm{C}_{44}=94.2 \mathrm{GPa}$ [14]. The anisotropy coefficient is $\mathrm{a}=2 \mathrm{C}_{44} /\left(\mathrm{C}_{11}-\mathrm{C}_{12}\right)=13.27$; the elastic behavior is highly anisotropic.

The estimation of the strain error with this setup is around $1.5 .10^{-4}$. Due to the elastic constants the normal stress error is then about $60 \mathrm{MPa}$ and the tangential stress error $30 \mathrm{MPa}$.

\subsection{Evolution of the crystallographic orientation of individual grains}

The position of the grains and their crystallographic orientations are plotted figure 4 . The circles represent the position of the CM. Among the 120 initial grains, we have only retained only those that we were able to follow during the whole loading cycle. Some grains were not always visible as they moved out of the beam when the specimen was strained. Finally, 101 grains fulfilled all conditions. Crystallographic orientations are well spread over the inverse pole figure and no particular texture was observed. During loading (figure 5) the grains rotate in various directions; the maximum rotation angle is about $2^{\circ}$; this value is of the same order of magnitude as the one measured on coarse grains [7].

The inverse rotation was observed during unloading. Considering the inverse pole figure, no particular influence of the initial orientation was noticed: two grains of similar orientation do not follow the same rotation path. Also the rotation magnitude differs when considering neighbored orientations. This shows that, even if the orientation is a key factor to study individual grain behavior, it does not explain all the differences observed.

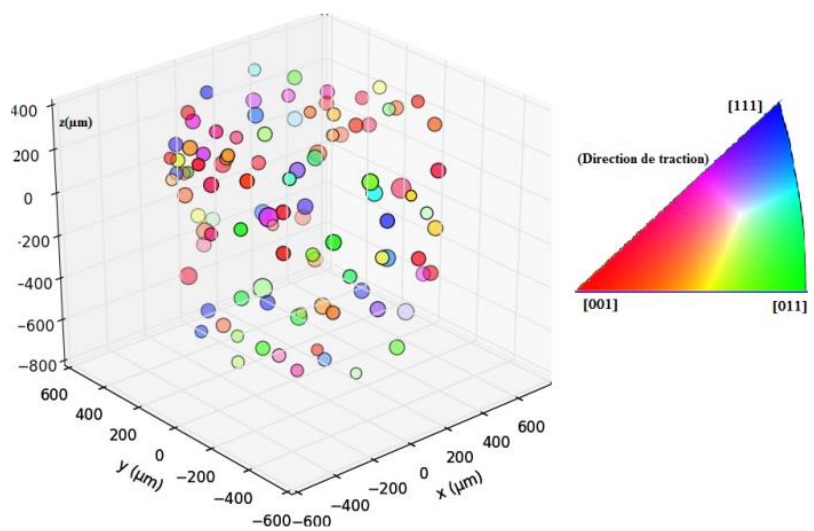

Figure 4. Crystallographic orientation (inverse pole figure in the tensile direction) and $\mathrm{CM}$ position of all individual grains at the initial state in the specimen. The tensile direction is parallel to the z-axis.

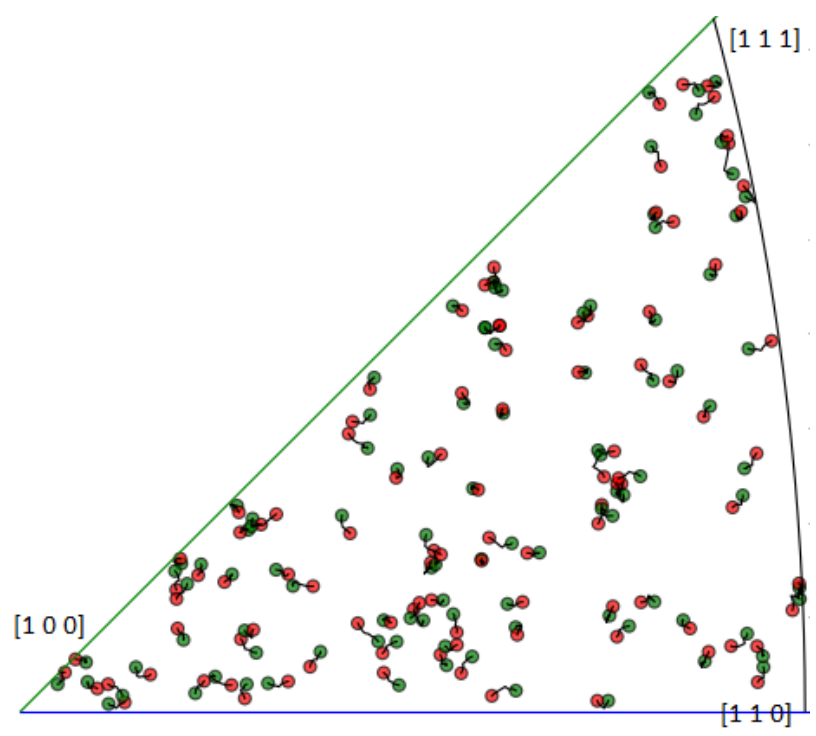

Figure 5. Evolution of the crystallographic orientation of the 101 grains during loading. Green dots : initial state. Red dots : final state.

\subsection{Distribution of individual grain strains and stresses}

Strain and stress tensors were determined for the 11 loading points. Figure 6 shows the values of strain $\left(\varepsilon_{11}\right)$ and stress $\left(\sigma_{11}\right)$ components in the tensile direction for the 101 grains and at the maximum macroscopic stress $(\Sigma=$ $540 \mathrm{MPa}$ ). These values concern only the austenite part of the grain whether it has transformed or not. Results are plotted in an inverse pole figure in the tensile direction.

A circle on the pole figure represents the initial orientation of the grain with respect to the tensile direction. Colors represent the elastic strain values. The 
applied macroscopic strain is 3\%; at this value, the alloy is no longer fully austenitic. Most of the grains have started to transform in martensite. Two regions can be identified on the inverse pole figure: on the right side, the strain values are much lower than on the left side.

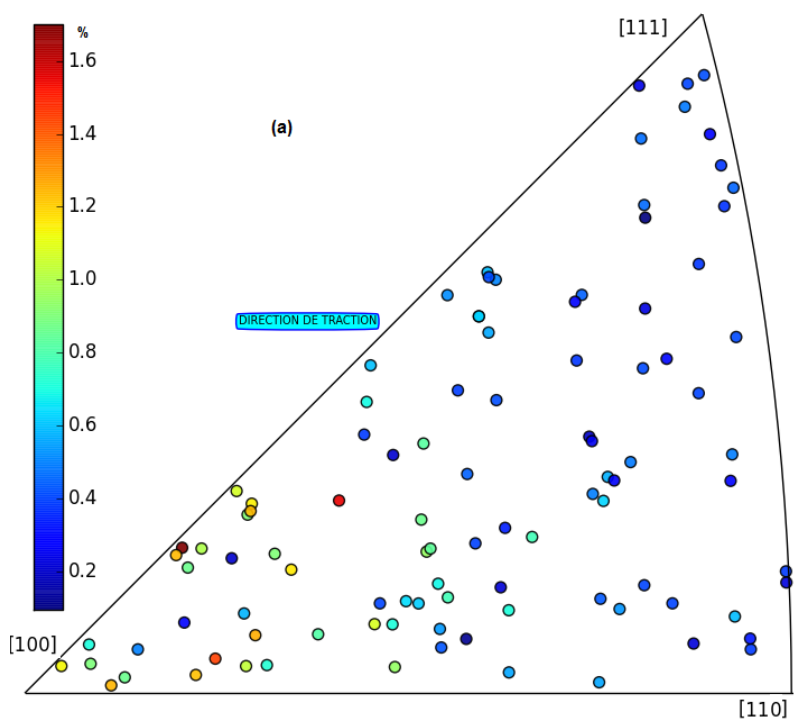

Figure 6. Distribution of elastic strain in the tensile direction as a function of initial crystallographic orientation of austenite at a macroscopic stress of 540MPa.

The strains are always lower than $0.5 \%$; this corresponds to grains for which the tensile direction is between [110] and [111]. For grains where the tensile direction is near the [100]-pole the strain values are higher than $0.6 \%$ and a large strain heterogeneity is observed in that zone: two grains have strain inferior to $0.4 \%$ whereas two others are above $1.2 \%$. [100]-oriented grains are known as soft grains that are easy to deform whereas [111]-oriented grains are hard grains in $\mathrm{Cu}$ based alloys $[1,3]$.

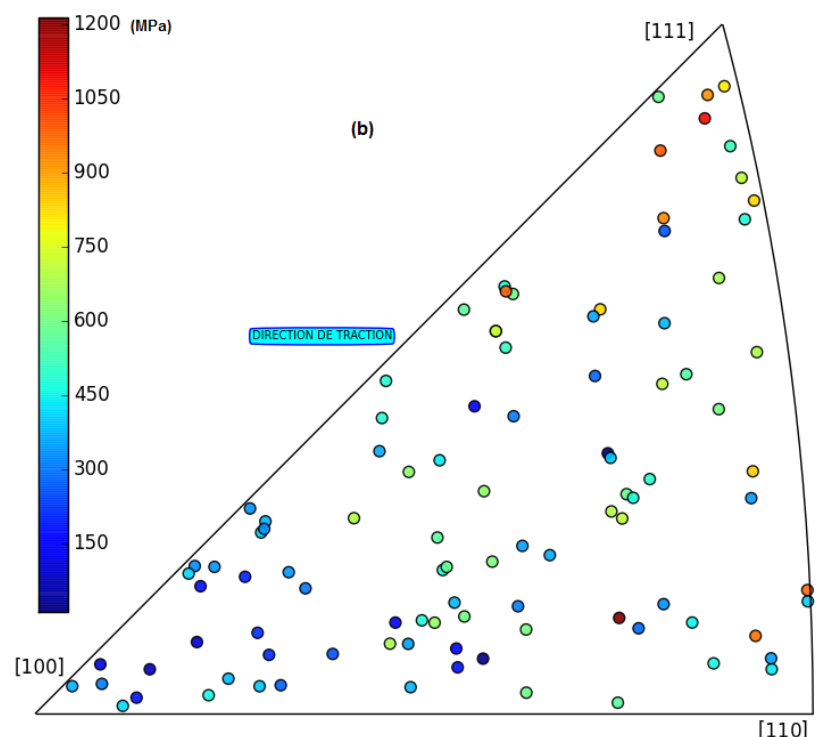

Figure 7. Distribution of stress in the tensile direction as a function of initial crystallographic orientation of austenite at a macroscopic stress of $540 \mathrm{MPa}$.
Stress values in the tensile direction are plotted figure 7 as a function of the crystallographic orientation of the grain, for the same loading point (macroscopic stress of $540 \mathrm{MPa}$, strain of $3 \%$ ). Colors represent the stress values.

Once again, one can observe two different zones on the inverse pole figure. However the tendency is inverted compared with strain distribution: the lowest values of stress are located near the [100]-pole and the distribution is quite homogeneous in that zone. The maximum value is around $400 \mathrm{MPa}$. In the rest of the pole figure, stress values are higher as well as the heterogeneity: several grains with similar orientations exhibit quite different stress states. For instance, near the [110]-pole, on the external border, one grain has an internal stress of 450 $\mathrm{MPa}$ and its nearest neighbor has a stress which is two times higher (around $900 \mathrm{MPa}$ ) while their orientations differ only slightly. This highlights once again that considering only the crystallographic orientation with respect to the tensile direction is not sufficient to predict individual behavior of grains in the polycrystal.

Some grains exhibit very high stress values (over $1200 \mathrm{MPa}$ ) but they are located at the limit of the illuminated zone. Therefore it is possible that the strains values are corrupted if their illuminated volume for diffraction changes varies during the experiment. This can induce some calculation error [2].

\subsection{Influence of grain positions on stress states}

In order to understand stress heterogeneities for a given orientation, we have plotted the stress values (in the tensile direction) of the grains as a function of the initial position of the center of mass. Figure 8 is a projection on the plane normal to the tensile axis; grains are represented according to their radial position in the specimen. Colors are in keeping with stress values. Only the component in the tensile direction is given for a macroscopic stress of $540 \mathrm{MPa}$.

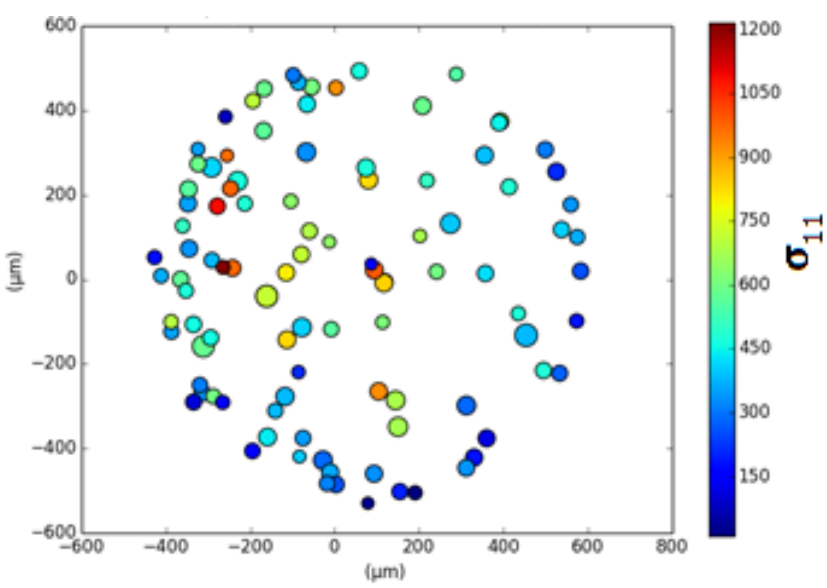

Figure 8. Positions of each of the grains in the cross section of the wire coloured by their stress values $\boldsymbol{\sigma}_{11}$ for the macroscopic load of 540MPa. 
It can be clearly seen that grains with a center of gravity near the external surface of the specimen are considerably less loaded than the grains in the bulk volume: most of the stress value are below $300 \mathrm{MPa}$ whereas the applied macroscopic stress is $540 \mathrm{MPa}$. Moreover, values are more homogeneous at the surface than in the volume: they vary between 300 and $1000 \mathrm{MPa}$. This difference is not related with particular crystallographic orientations as no texture was observed (see figure 4); considering the pairs of grains mentioned in section 3.2 with similar orientation but very different stress, we observed that the less stressed grains are located at the free surface while the most stressed are embedded in the volume. This highlights the role of the free surface as a key factor on the mechanical behavior of individual grains.

\subsection{Internal stress evolution}

Figure 9 shows a comparison between the applied macroscopic stress and the internal stress arithmetic averages $\left(<\boldsymbol{\sigma}_{11}>,<\boldsymbol{\sigma}_{22}>\right.$ and $\left\langle\boldsymbol{\sigma}_{33}>\right)$ for the first cycle. The $\sigma_{11}$ component is parallel the tensile direction.

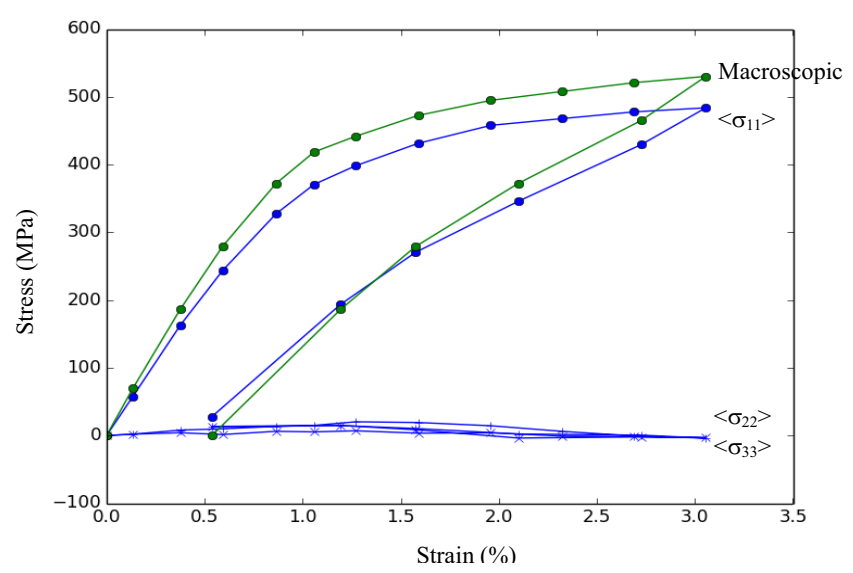

Figure 9. Applied stress (green) and internal stresses arithmetic averages (blue) in the hundred austenite grains versus the macroscopic strain.

We first note that the average values of $\sigma_{22}$ and $\sigma_{33}$ are small while this is not the case for all individual values. This is quite logical because we applied a uniaxial tensile loading perpendicular to the « $2 »$ and « $3 »$ directions.

The average of the internal stresses $\sigma_{11}$ in the tensile direction follows the same evolution as the applied macroscopic stress. A slight difference between the curves is observed during loading for macroscopic stresses higher than $300 \mathrm{MPa}$ and until unloading at a stress of $300 \mathrm{MPa}$. This means that the shift is observed in the transformation region where austenite and martensite co-exist. This shows the coherence of the determined values: using 3DXRD, we only measured stress in the austenite phase. So, in the elastic domain, the average measured stresses are equal to the applied one; when martensite appears, a stress redistribution between both phases occur. As the austenite stress is lower than the macroscopic one, this indicates that martensite must be harder than austenite, with a higher internal stress than the macroscopic one.

\section{CONCLUSIONS}

The 3DXRD method allowed us to obtain experimental results for over one hundred grains (mesoscopic) during a superelastic cycle in a SMA. These results help to understand the interactions between grains during the martensitic transformation depending on their orientation, their size, their position and their environment in the tensile specimen. It was shown that the free surface has important effects on the mechanical behaviour. A strong stress heterogeneity was observed in the polycrystal, with values varying by a factor of more than 3 .

This method can be supplemented with tomographic measurements to get the shape of the grains and offers unique insights into the mechanical behavior of many materials and specifically SMA.

\section{REFERENCES}

1. P. Sittner, P. Lukas, D. Neov, MR. Daymond, V. Novak, GM. Swallowe, Mater Sci Eng A324, 225 (2002)

2. K. Otsuka, C. Wayman, H. Sakamoto, K. Shimizu, Acta. Met. 24, 207 (1976)

3. Kaouache B, Berveiller S, Inal K, Eberhardt A, Patoor E. Mater Sci. Eng. A378, 232 (2004)

4. S.F. Nielsen, E.M. Lauridsen, D. Juul Jensen, H.F. Poulsen, Mat. Sci. Eng. A319-312, 179 (2001)

5. L. Margulies, T. Lorentzen, H.F. Poulsen, T. Leffers, Acta. Mat. 50, 1771 (2002)

6. H.F. Poulsen, L. Margulies, S. Schmidt, G. Winther, Acta. Mat. 51, 3821 (2003)

7. S. Berveiller, B. Malard, J. Wright, E. Patoor, G. Geandier, Acta. Mat. 59, 3636 (2011)

8. S.E. Offerman, N.H. Van Dijk, J. Siestma, E.M. Lauridsen, L. Margulies, S. Grigull, H.F. Poulsen, S. van der Zwaag, Nuclear Instr. Meth. Physics Res. B238, 107 (2005)

9. P. Hedstrom, U. Lienert, J. Almer, M. Oden, Mat. Letters 62, 338 (2008)

10. E.M. Lauridsen, S. Schmidt, H.F. Poulsen, R.M. Suter, J. Appl. Cryst. 34(6), 744 (2001)

11. H.F. Poulsen, S.F. Nielsen, E.M. Lauridsen, S. Schmidt, R.M. Suter, U. Lienert, J. Appl. Cryst. 34(6), 751 (2001)

12. http://fable.wiki.sourgeforge.net/imaged11

13. J. Oddershede, S. Schmidt, H. F. Poulsen, H. O. Sørensen, J. Wright and W. Reimers, J. Appl. Cryst. 43, 539-549 (2010)

14. S. Belkahla, Doctorate Thesis, INSA Lyon (1990) 
